\title{
REMARKS ON NONLINEAR BIHARMONIC EVOLUTION EQUATION OF KIRCHHOFF TYPE IN NONCYLINDRICAL DOMAIN
}

\author{
J. LÍMACO, H. R. CLARK, and L. A. MEDEIROS
}

Received 24 June 2002

\begin{abstract}
We investigate a boundary value problem for a nonlinear evolution biharmonic operator motivated by flexion of fully clamped beam in two different physical situations. In the first, the supports of the ends of the beam are fixed and in the second one, the supports of the ends of the beam have small displacements.
\end{abstract}

2000 Mathematics Subject Classification: 35K55, 35B40.

1. Introduction. In this paper, we investigate existence and asymptotic behavior for a boundary value problem for the operator

$$
\mathscr{L} u=a(x) u^{\prime \prime}+\Delta(b(x) \Delta u)-\widehat{M}\left(x, t, \int_{D}|\nabla u(x, t)|^{2} d x\right) \Delta u
$$

motivated by the problem of vertical flexion of fully clamped beams. Our main goal in this paper is to study the properties previously mentioned when the set $D$ is a time-dependent nonempty bounded subset $\Omega_{t}$ of $\mathbb{R}^{n}$, which means that the supports of the extremes of the beam have small displacements. Note that the sets $\Omega_{t}$, where $u=u(x, t)$ is defined, change with the time, which yields a noncylindrical domain

$$
\hat{Q}=\bigcup_{0 \leq t<\infty} \Omega_{t} \times\{t\}
$$

where we formulate the moving boundary value problem. To reach these objectives, we initially study a mixed problem associated with the operator $\mathscr{L} u$ when the set $D$ is considered a nonempty bounded open subset $\Omega$ of $\mathbb{R}^{n}$, that is, when the supports of the extremes of the beam are fixed.

The method we will employ to solve the initial value problem with moving boundary was idealized by Lions [8], see also Lions [9, Chapter 3, page 413] for the operator of the type $u \rightarrow u^{\prime \prime}-\Delta u+|u|^{\rho} u$. It consists in transforming the boundary value problem formulated for the operator $\mathscr{L} u$ in a noncylindrical domain $\widehat{Q}$ into a cylindrical problem for a perturbed operator obtained from 
$\mathscr{L} u$, whose perturbed operator depends on a parameter $0<\epsilon<1$ destined to tend to zero. This method was idealized and called penalty method by Lions [8]. It has its origin in the calculus of variations with vinculums, see Lions [9, Chapter 3].

The penalty method was also employed by Cooper-Bardos [2] for the same operator $u \rightarrow u^{\prime \prime}-\Delta u+|u|^{\rho} u$ for noncylindrical domains $\hat{Q}$, but which are "time like" instead of increasing as in Lions [8]. In Medeiros [11, 12], it is considered the operator $u \rightarrow u^{\prime \prime}-\Delta u+F(u)$ by penalty method in increasing domains, see also Strauss [6], Cooper-Medeiros [3], Inoue [7], Nakao-Narazaki [14], and Rabello [5]. We employ certain techniques for cylindrical domains as in Hosoya and Yamada [4].

The penalty method reduces the noncylindrical problem into a cylindrical one. For this reason, we open Section 3, studying with certain details, the boundary value problem for the biharmonic evolution operator in a cylinder $Q$. In Section 2, we fix part of the notations.

2. Notations. The scalar product and norm in $L^{2}(\Omega)$ and $H_{0}^{1}(\Omega)$ are, respectively, represented by $(\cdot, \cdot),((\cdot, \cdot)),|\cdot|$, and $\|\cdot\|$, where $\Omega$ is an open bounded set of $\mathbb{R}^{n}$. We represent by $|u(x, t)|$ the absolute value of the real number $u(x, t)$ and $|\nabla u(x, t)|$ the norm in $\mathbb{R}^{n}$ of the vector $\nabla u(x, t)$. When we write $|u(t)|,|\nabla u(t)|,|\Delta u(t)|$, and $\|u(t)\|$, we mean the $L^{2}(\Omega)$ norm of $u(x, t)$, $\nabla u(x, t)$, and $\Delta u(x, t)$ and the $H_{0}^{1}(\Omega)$ norm of $u(x, t)$. Let $(x, t)$ be a point of $\mathbb{R}^{n} \times[0, \infty[$. Thus, we consider the noncylindrical domains

$$
\left.\hat{Q} \subset \mathbb{R}^{n} \times\right] 0, \infty\left[\quad \text { or } \quad \hat{Q} \subset \mathbb{R}^{n} \times\right] 0, T[\text {, with } T>0 \text {, }
$$

where $\Omega_{s}$ represents the sections of $\hat{Q} \cap\{t=s\}$, for $0 \leq s \leq T, \Gamma_{s}$ is the boundary of $\Omega_{s}$, and the lateral boundary of $\hat{Q}$ is given by $\Sigma_{s}=\bigcup_{0<s<T} \Gamma_{s}$. The boundary of $\hat{Q}$ is given by $\partial \hat{Q}=\Omega_{0} \cup \Sigma_{T} \cup \Omega_{T}$. We suppose that $\Omega_{0} \subset \Omega$ and $\hat{Q}$ is contained in a cylinder $Q=\Omega \times] 0, T$, for $0<t<T$. In Section 4 , we will fix a fundamental regularity condition on $\hat{Q}$.

The spaces $L^{2}\left(\Omega_{t}\right), H_{0}^{1}\left(\Omega_{t}\right)$, and $H^{2}\left(\Omega_{t}\right)$ are identified to subspaces of $L^{2}(\Omega)$, $H_{0}^{1}(\Omega)$, and $H^{2}(\Omega)$. Thus, we define $L^{p}\left(0, T ; L^{2}\left(\Omega_{t}\right)\right)$, for $1 \leq p \leq \infty$, as the space of functions $v \in L^{2}\left(0, T ; L^{2}\left(\Omega_{t}\right)\right)$ such that $v(t) \in L^{2}\left(\Omega_{t}\right)$ almost everywhere. By this method we also define $L^{p}\left(0, T ; H_{0}^{1}\left(\Omega_{t}\right)\right)$ and $L^{p}\left(0, T ; H_{0}^{2}\left(\Omega_{t}\right)\right)$.

The real function $M(x, t, \lambda)$ is defined by $(x, t) \in Q$ and $0<\lambda<\infty$. The regularity on $M$ will be fixed in Section 3 . Finally, by $\widehat{M}(x, t, \lambda)$ we represent the restriction of $M(x, t, \lambda)$ to the noncylindrical domain $\widehat{Q}$.

\section{Cylindrical domain}

3.1. Weak solutions. According to the notation of Section 2, we represent by $Q$ the cylinder $\Omega \times] 0, T\left[\right.$ of $\mathbb{R}^{n}$, for $T>0$, with the lateral boundary 
$\Sigma=\Gamma \times] 0, T[$. In these conditions, we consider the boundary value problem

$$
\begin{gathered}
a(x) u^{\prime \prime}(x, t)+\Delta(b(x) \Delta u(x, t))-M\left(x, t, \int_{\Omega}|\nabla u(x, t)|^{2} d x\right) \Delta u(x, t) \\
+\delta u^{\prime}(x, t)=0 \quad \text { in } Q \\
u(x, t)=\frac{\partial u}{\partial v}(x, t)=0 \quad \text { on } \Sigma \\
u(x, 0)=u_{0}(x), \quad u^{\prime}(x, 0)=u_{1}(x) \quad \text { in } \Omega
\end{gathered}
$$

where $v$ is the exterior unit normal vector to $\Sigma, \partial / \partial v$ the normal derivative, and $\delta$ a positive real parameter. By $u=u(x, t)$ we represent a real function defined for $(x, t) \in Q$.

On the functions of system (3.1), we assume the hypothesis

$$
a(x), b(x) \in L^{\infty}(\Omega), \quad \text { with } 0<a_{0}<a(x)<1,0<b_{0}<b(x)<b_{1} .
$$

The function $M(x, t, \lambda)$ is a $C^{1}$-real function in the variables $x \in \Omega, t \geq 0, \lambda \geq 0$, where

$$
\begin{gathered}
M(x, t, \lambda) \geq 0, \quad\left|\frac{\partial M}{\partial \lambda}\right| \leq C_{1}|\lambda|^{p-1} \\
\left|\nabla_{x} M\right| \leq C_{2}|\lambda|^{p}, \quad\left|\frac{\partial M}{\partial t}\right| \leq C_{3}|\lambda|^{p} \quad \text { for } p \geq 1 .
\end{gathered}
$$

DEFINITION 3.1. A weak solution of the boundary value problem (3.1) is called a real function $u=u(x, t)$, defined for $(x, t) \in Q$ such that

$$
u \in L^{2}\left(0, T ; H_{0}^{2}(\Omega)\right), \quad u^{\prime} \in L^{2}\left(0, T ; L^{2}(\Omega)\right), \quad \text { for } T>0,
$$

satisfying the identity integral

$$
\begin{aligned}
-\int_{0}^{T} \int_{\Omega} a(x) u^{\prime}(x, t) v(x) \theta^{\prime}(t) d x d t \\
\quad+\int_{0}^{T} \int_{\Omega} b(x) \Delta u(x, t) \Delta v(x) \theta(t) d x d t \\
\quad+\int_{0}^{T} \int_{\Omega} M\left(x, t,\|u(t)\|^{2}\right) \nabla u(x, t) \cdot \nabla v(x) \theta(t) d x d t \\
\quad+\int_{0}^{T} \int_{\Omega} \nabla_{x} M\left(x, t,\|u(t)\|^{2}\right) \cdot \nabla u(x, t) v(x) \theta(t) d x d t \\
\quad+\delta \int_{0}^{T} \int_{\Omega} u^{\prime}(x, t) v(x) \theta(t) d x d t=0 \quad \forall v \in H_{0}^{2}(\Omega), \theta \in \mathscr{D}(0, T),
\end{aligned}
$$

and the initial conditions

$$
u(x, 0)=u_{0}(x), \quad u^{\prime}(x, 0)=u_{1}(x) .
$$

Note that $\mathscr{D}(0, T)$ is the space of $C^{\infty}$-functions with compact support in ] $0, T[$. 
THEOREM 3.2. Supposing $u_{0} \in H_{0}^{2}(\Omega), u_{1} \in L^{2}(\Omega)$, and

$$
C_{6} H^{p}(0)<\frac{\delta b_{0}}{10},
$$

where the function $H(t)$ and the constant $C_{6}$ are defined in (3.21) and (3.31), respectively, then there exists only one real function $u=u(x, t)$ defined for all $(x, t) \in Q$, solution of the boundary value problem (3.1) in the sense of Definition 3.1.

PROOF. We employ Faedo-Galerkin approximate method with a Hilbertian basis $\left(w_{j}\right)_{j \in \mathbb{N}}$ of the Sobolev space $H_{0}^{2}(\Omega)$ (cf. Brezis [1]). If $V_{m}$ is the subspace of $H_{0}^{2}(\Omega)$ spanned by the $m$ first vectors $\left\{w_{1}, w_{2}, w_{3}, \ldots, w_{n}\right\}$, we will formulate the approximate problem as it proceeds.

Find a function

$$
u_{m}(x, t)=\sum_{j=1}^{m} g_{j m}(t) w_{j}(x)
$$

in $V_{m}$ solution of the following initial value problem:

$$
\begin{gathered}
\left(a u_{m}^{\prime \prime}(t), w\right)+\left(b \Delta u_{m}(t), \Delta w\right)+\left(M\left(t,\|u(t)\|^{2}\right) \nabla u_{m}(t), \nabla w\right) \\
+\left(\nabla_{x} M\left(t,\left\|u_{m}(t)\right\|^{2}\right) \cdot \nabla u_{m}(t), w\right)+\delta\left(u_{m}^{\prime}(t), w\right)=0, \\
u_{m}(x, 0)=u_{0 m}(x) \longrightarrow u_{0}(x) \quad \text { in } H_{0}^{2}(\Omega), \\
u_{m}^{\prime}(x, 0)=u_{1 m}(x) \longrightarrow u_{1}(x) \quad \text { in } L^{2}(\Omega),
\end{gathered}
$$

for all $w \in V_{m}$. There exists a local solution $u_{m}=u_{m}(x, t)$ of (3.9) on the interval $\left[0, t_{m}\right.$ [. The estimates that follow permit to extend the solution $u_{m}$ to the interval [0, $T$ [ for all $T>0$ and to take the limit in (3.9).

Henceforth, in order to make the notation better, we will write $u$ instead of $u_{m}$.

ESTImATE 1. Setting $w=u^{\prime}$ into (3.9) and using the hypothesis (3.3) yield

$$
\begin{aligned}
& \left(a u^{\prime \prime}(t), u^{\prime}(t)\right)=\frac{1}{2} \frac{d}{d t} \int_{\Omega} a(x)\left|u^{\prime}(x, t)\right|^{2} d x \\
& \left(b \Delta u(t), \Delta u^{\prime}(t)\right)=\frac{1}{2} \frac{d}{d t} \int_{\Omega} b(x)|\Delta u(x, t)|^{2} d x \\
& \int_{\Omega} M\left(x, t,\|u(t)\|^{2}\right) \frac{d}{d t}|\nabla u(x, t)|^{2} d x \\
& =\frac{d}{d t}\left[\int_{\Omega} M\left(x, t,\|u(t)\|^{2}\right)|\nabla u(x, t)|^{2} d x\right] \\
& \quad-\int_{\Omega} \frac{\partial}{\partial t} M\left(x, t,\|u(t)\|^{2}\right)|\nabla u(x, t)|^{2} d x \\
& \quad-2\left[\int_{\Omega} \frac{\partial}{\partial \lambda} M\left(x, t,\|u(t)\|^{2}\right)|\nabla u(x, t)|^{2} d x\right]\left(\nabla u^{\prime}(t), \nabla u(t)\right)
\end{aligned}
$$




$$
\begin{aligned}
& \left|\int_{\Omega}\left[\nabla_{x} M\left(x, t,\|u(t)\|^{2}\right) \cdot \nabla u(x, t)\right] u^{\prime}(x, t) d x\right| \\
& \quad \leq C_{2}|| u(t)\left\|^{2 p} \int_{\Omega}|\nabla u(x, t)|\left|u^{\prime}(x, t)\right| d x \leq C_{2}\right\| u(t) \|^{2 p+1}\left|u^{\prime}(t)\right|,
\end{aligned}
$$

where we have used in the last step of (3.13) both Cauchy-Schwarz and Sobolev inequalities. The two last integrals of the right-hand side of (3.12) can be estimated by

$$
\begin{aligned}
& \left.\left.\left|\int_{\Omega} \frac{\partial}{\partial \lambda} M\left(x, t,\|u(t)\|^{2}\right)\right| \nabla u(x, t)\right|^{2} d x\left|\leq C_{1}\|u(t)\|^{2 p-2} \int_{\Omega}\right| \nabla u(x, t)\right|^{2} d x, \\
& \left.\left.\left|\int_{\Omega} \frac{\partial}{\partial t} M\left(x, t,\|u(t)\|^{2}\right)\right| \nabla u(x, t)\right|^{2} d x\left|\leq C_{3}\|u(t)\|^{2 p} \int_{\Omega}\right| \nabla u(x, t)\right|^{2} d x .
\end{aligned}
$$

Thus, after setting $w=u^{\prime}$ into (3.9) and considering the expressions from (3.10) to (3.15), we have

$$
\begin{aligned}
\frac{1}{2} \frac{d}{d t}\left[\int _ { \Omega } \left(a(x)\left|u^{\prime}(x, t)\right|^{2}+b(x)|\Delta u(x, t)|^{2}\right.\right. \\
\left.\left.\quad+2 M\left(x, t,\|u(t)\|^{2}\right)|\nabla u(x, t)|^{2}\right) d x\right]+\delta\left|u^{\prime}(t)\right|^{2} \\
\quad \leq C_{1}|| u(t)\left\|^{2 p}|\Delta u(t)|\left|u^{\prime}(t)\right|+C_{2}|| u(t)\right\|^{2 p+1}\left|u^{\prime}(t)\right|+C_{3}|| u(t) \|^{2 p+2} \\
\quad \leq C_{4}|\Delta u(t)|^{2 p+1}\left|u^{\prime}(t)\right|+C_{3}|\Delta u(t)|^{2 p+2},
\end{aligned}
$$

where we have used the inequality $\|u(t)\|^{2} \leq C_{0}|\Delta u(t)|^{2}$ for all $u \in H_{0}^{2}(\Omega)$ in the last step of (3.16).

ESTIMATE 2. Setting $w=u$ into (3.9), we get

$$
\begin{gathered}
\left(a u^{\prime \prime}(t), u(t)\right)=\frac{d}{d t} \int_{\Omega} a(x) u^{\prime}(x, t) u(x, t) d x \\
\quad-\int_{\Omega} a(x)\left|u^{\prime}(x, t)\right|^{2} d x, \\
\left|\int_{\Omega}\left[\nabla_{x} M\left(x, t,\|u(t)\|^{2}\right) \cdot \nabla u(x, t)\right] u(x, t) d x\right| \\
\quad \leq C_{2}|| u(t) \|^{2 p} \int_{\Omega}|\nabla u(x, t)||u(x, t)| d x \\
\leq C_{2}|| u(t) \|^{2 p+1}|u(t)| \leq C_{5}|\Delta u(t)|^{2 p+2},
\end{gathered}
$$

where we have used in (3.18) the hypothesis (3.3), both Cauchy-Schwarz and Sobolev's inequalities and $\|u(t)\|^{2} \leq C_{0}|\Delta u(t)|^{2}$ for all $u \in H_{0}^{2}(\Omega)$. 
Thus, after setting $w=u$ into (3.9) and taking (3.17) and (3.18) into account, we obtain

$$
\begin{aligned}
& \frac{d}{d t} \int_{\Omega} a(x) u^{\prime}(x, t) u(x, t) d x-\int_{\Omega} a(x)\left|u^{\prime}(x, t)\right|^{2} d x \\
& \quad+\int_{\Omega}\left(b(x)|\Delta u(x, t)|^{2}+M\left(x, t,\|u(t)\|^{2}\right)|\nabla u(x, t)|^{2}\right) d x \\
& \quad+\frac{\delta}{2} \frac{d}{d t} \int_{\Omega}|u(x, t)|^{2} d x \leq C_{5}|\Delta u(t)|^{2 p+2} .
\end{aligned}
$$

Multiplying (3.19) by $\delta / 4$ and summing to (3.16) yield

$$
\begin{gathered}
\frac{1}{2} \frac{d}{d t}\left[\int _ { \Omega } \left(a(x)\left|u^{\prime}(x, t)\right|^{2}+b(x)|\Delta u(x, t)|^{2}+\frac{\delta}{2} a(x) u^{\prime}(x, t) u(x, t)\right.\right. \\
\left.\left.\quad+\frac{\delta^{2}}{4}|u(x, t)|^{2}+2 M\left(x, t,\left.|| u(t)\right|^{2}\right)|\nabla u(x, t)|^{2}\right) d x\right] \\
+\delta \int_{\Omega}\left|u^{\prime}(x, t)\right|^{2} d x+\frac{\delta}{4} \int_{\Omega}\left(-a(x)\left|u^{\prime}(x, t)\right|^{2}+b(x)|\Delta u(x, t)|^{2}\right. \\
\left.+M\left(x, t,\|u(t)\|^{2}\right)|\nabla u(x, t)|^{2}\right) d x \\
\leq C_{4}|\Delta u(t)|^{2 p+1}\left|u^{\prime}(t)\right|+C_{3}|\Delta u(t)|^{2 p+2}+\frac{C_{5} \delta}{4}|\Delta u(t)|^{2 p+2} .
\end{gathered}
$$

Denoting by $H(t)=H(u(t))$ the function

$$
\begin{aligned}
H(t)=\frac{1}{2} \int_{\Omega}( & a(x)\left|u^{\prime}(x, t)\right|^{2}+b(x)|\Delta u(x, t)|^{2} \\
& +\frac{\delta}{2} a(x) u^{\prime}(x, t) u(x, t)+\frac{\delta^{2}}{4}|u(x, t)|^{2} \\
& \left.+2 M\left(x, t,\|u(t)\|^{2}\right)|\nabla u(x, t)|^{2}\right) d x
\end{aligned}
$$

and using the hypothesis $a(x) \leq 1$, we get from usual inequality that

$$
\begin{aligned}
\left|\frac{\delta}{4} \int_{\Omega} a(x) u^{\prime}(x, t) u(x, t)\right| & \leq \frac{\delta}{4} \int_{\Omega} a(x)\left|u^{\prime}(x, t)\right||u(x, t)| d x \\
& \leq \frac{1}{4} \int_{\Omega} a(x)\left|u^{\prime}(x, t)\right|^{2} d x+\frac{\delta^{2}}{16} \int_{\Omega}|u(x, t)|^{2} d x .
\end{aligned}
$$

Hence we have

$$
\frac{\delta}{4} \int_{\Omega} a(x) u^{\prime}(x, t) u(x, t) \geq-\frac{1}{4} \int_{\Omega} a(x)\left|u^{\prime}(x, t)\right|^{2} d x-\frac{\delta^{2}}{16} \int_{\Omega}|u(x, t)|^{2} d x .
$$


Substituting (3.23) into (3.21), we obtain

$$
\begin{aligned}
H(t) \geq \int_{\Omega}( & \frac{1}{4} a(x)\left|u^{\prime}(x, t)\right|^{2}+\frac{1}{2} b(x)|\Delta u(x, t)|^{2} \\
& \left.+\frac{\delta^{2}}{16}|u(x, t)|^{2}+2 M\left(x, t,\left.|| u(t)\right|^{2}\right)|\nabla u(x, t)|^{2}\right) d x \\
\geq & \min \left\{\frac{1}{4} a_{0}, \frac{1}{2} b_{0}\right\} \int_{\Omega}\left(\left|u^{\prime}(x, t)\right|^{2}+|\Delta u(x, t)|^{2}\right) d x \geq 0,
\end{aligned}
$$

where we have used in the last step of (3.24) the hypotheses (3.2) and (3.3).

Modifying both the sixth and the seventh terms of the left-hand side of (3.20) and using the hypothesis (3.2), we get

$$
\begin{aligned}
\delta \int_{\Omega}\left(\left|u^{\prime}(x, t)\right|^{2}-\frac{1}{4} a(x)\left|u^{\prime}(x, t)\right|^{2}\right) d x & \\
& \geq \frac{\delta}{4} \int_{\Omega}(1-a(x))\left|u^{\prime}(x, t)\right|^{2} d x \geq 0 .
\end{aligned}
$$

Thus, from (3.20), (3.21), (3.25), and hypothesis (3.2), we obtain

$$
\begin{aligned}
\frac{d}{d t} H(t)+ & |\Delta u(t)|^{2}\left(\frac{\delta b_{0}}{8}-C_{3}|\Delta u(t)|^{2 p}-C_{4}|\Delta u(t)|^{2 p-1}\left|u^{\prime}(t)\right|\right. \\
& \left.-\frac{C_{5} \delta}{4}|\Delta u(t)|^{2 p}\right)+\frac{\delta b_{0}}{8}|\Delta u(t)|^{2} \\
+ & \frac{3 \delta}{4}\left|u^{\prime}(t)\right|^{2}+\frac{\delta}{4} \int_{\Omega} M\left(x, t,\|u(t)\|^{2}\right)|\nabla u(x, t)|^{2} d x \leq 0 .
\end{aligned}
$$

We define $\gamma(t)=\gamma(u(t))$ by

$$
\gamma(t)=C_{3}|\Delta u(t)|^{2 p}+C_{4}|\Delta u(t)|^{2 p-1}\left|u^{\prime}(t)\right|+\frac{C_{5} \delta}{4}|\Delta u(t)|^{2 p} .
$$

Thus, (3.26) and (3.27) yield

$$
\frac{d}{d t} H(t)+|\Delta u(t)|^{2}\left(\frac{\delta b_{0}}{8}-\gamma(t)\right) \leq 0
$$

From (3.24), we can write

$$
|\Delta u(t)| \leq \sqrt{\frac{2}{b_{0}}} \sqrt{H(t)}, \quad\left|u^{\prime}(t)\right| \leq \frac{2}{\sqrt{a_{0}}} \sqrt{H(t)} .
$$

Then, from (3.27) and (3.29), we obtain

$$
\gamma(t) \leq C_{6} H^{p}(t) \quad \forall t \geq 0,
$$

where

$$
C_{6}=\frac{2}{b_{0}}+C_{4}\left(\frac{2}{b_{0}}\right)^{p-1 / 2}\left(\frac{2}{\sqrt{a_{0}}}\right)+\frac{C_{0} \delta}{4} .
$$


Our next task is to show that $H(t)$ is bounded for all $t \geq 0$. In fact, if $\gamma(t) \leq$ $\delta b_{0} / 8$ for all $t \geq 0$, it follows from (3.28) that $(d / d t) H(t) \leq 0$, and hence, $H(t) \leq H(0)$ for all $t \geq 0$. This gives global estimates for the approximate solutions, which is sufficient to take the limits in system (3.9).

Otherwise we have $\gamma(t)>\delta b_{0} / 8$ for some $t>0$, and thus, we will prove that this hypothesis imply a contradiction. In fact, from (3.7) and (3.30), $\gamma(0)<$ $\delta b_{0} / 10$. As the function $\gamma(t)$ is continuous for $t>0$, there exists $t^{*}>0$ such that $t^{*}=\min \left\{t>0 ; \gamma(t)=\delta b_{0} / 8\right\}$. Thus, we have

$$
\begin{gathered}
\gamma(t)<\frac{\delta b_{0}}{8} \quad \text { if } 0 \leq t<t^{*}, \\
\gamma\left(t^{*}\right)=\frac{\delta b_{0}}{8}
\end{gathered}
$$

From (3.28) and (3.32a), we have $H(t) \leq H(0)$ for all $0 \leq t \leq t^{*}$. Therefore, $H\left(t^{*}\right) \leq H(0)$. From (3.7) and (3.30), we have

$$
\gamma\left(t^{*}\right) \leq C_{6} H^{p}\left(t^{*}\right) \leq C_{6} H^{p}(0)<\frac{\delta b_{0}}{10}<\frac{\delta b_{0}}{8},
$$

which is a contradiction with (3.32b). It then follows that

$$
\begin{gathered}
0<\gamma(t)<\frac{\delta b_{0}}{8} \quad \forall t \geq 0, \\
H(t) \leq H(0) \leq\left(\frac{\delta b_{0}}{10 C_{3}}\right)^{1 / p} \quad \forall 0 \leq t, \text { including when } t \rightarrow \infty .
\end{gathered}
$$

Hence, returning to the notation $u=u_{m}(x, t)$ we obtain from (3.24) the global estimate in $t$ for $u_{m}(x, t)$ given by

$$
\left|u_{m}^{\prime}(t)\right|^{2}+\left|\Delta u_{m}(t)\right|^{2} \leq C_{7} \quad \forall t \geq 0
$$

Observing that $\left\|u_{m}\right\|^{2} \leq C_{0}\left|\Delta u_{m}\right|^{2}$ for all $u_{m} \in H_{0}^{2}(\Omega)$, and from Aubin-Lions' theorem (cf. Lions [9]), we obtain a subsequence, still represented by $\left(u_{m}\right)_{m \in \mathbb{N}}$, such that $u_{m} \rightarrow u$ strongly in $L^{2}\left(0, T ; H_{0}^{1}(\Omega)\right)$ and almost everywhere in $Q$. Therefore, we can take the limit as $m \rightarrow \infty$ for this subsequence in the approximate problem (3.9) and obtain the proof of Theorem 3.2.

3.2. Exponential decay. Our goal is to show that the total energy of system (3.1) decays exponentially as $t \rightarrow+\infty$. Thus, we will initially show the exponential decay of the energy associated with the approximate solutions $u_{m}$. The estimates obtained in (3.36) allows us to conclude the same result for the solutions $u$. Here, we also write $u$ instead of $u_{m}$. Thus, we have the following theorem. 
THEOREM 3.3. Assuming all the hypotheses of Theorem 3.2, then the energy $E(t)$ of system (3.1) satisfies

$$
E(t) \leq 2 K_{4} \exp \left\{-K_{3} t\right\} \quad \forall t \geq 0,
$$

where

$$
\begin{aligned}
E(t)=\frac{1}{2} \int_{\Omega}( & \left(a(x)\left|u^{\prime}(x, t)\right|^{2}+b(x)|\Delta u(x, t)|^{2}\right. \\
& \left.+2 M\left(x, t,\|u(t)\|^{2}\right)|\nabla u(x, t)|^{2}\right) d x,
\end{aligned}
$$

$K_{3}$ and $K_{4}$ are real positive constants defined in (3.43) and (3.44), respectively.

Proof. From (3.26), (3.27), and (3.34), we have

$$
\begin{aligned}
\frac{d}{d t} H(t)+\frac{\delta}{4} \int_{\Omega}( & \left|u^{\prime}(x, t)\right|^{2}+\frac{b_{0}}{2}|\Delta u(x, t)|^{2} \\
& \left.+M\left(x, t,\|u(t)\|^{2}\right)|\nabla u(x, t)|^{2}\right) d x \leq 0 .
\end{aligned}
$$

Denoting by $K_{1}=(\delta / 4) \min \left\{1, b_{0} / 2\right\}$, we can write, from (3.39),

$$
\begin{aligned}
\frac{d}{d t} H(t)+K_{1} \int_{\Omega} & \left(\left|u^{\prime}(x, t)\right|^{2}+|\Delta u(x, t)|^{2}\right. \\
& \left.+M\left(x, t,\|u(t)\|^{2}\right)|\nabla u(x, t)|^{2}\right) d x \leq 0 .
\end{aligned}
$$

On the other hand, from the definition of $H$ in (3.21), hypothesis (3.2), inequality $|u(t)|^{2} \leq C_{0}|\Delta u(t)|^{2}$ for all $H_{0}^{2}(\Omega)$, and usual inequalities, we obtain

$$
\begin{aligned}
H(t) \leq \frac{1}{2} \int_{\Omega}( & \left|u^{\prime}(x, t)\right|^{2}+b_{1}|\Delta u(x, t)|^{2}+\frac{\delta}{4}|u(x, t)|^{2} \\
& \left.+\frac{\delta}{4}\left|u^{\prime}(x, t)\right|^{2}+\frac{\delta^{2}}{4}|u(x, t)|^{2}+2 M\left(x, t, \|\left. u(t)\right|^{2}\right)|\nabla u(x, t)|^{2}\right) d x \\
=\frac{1}{2} \int_{\Omega}[ & \left(1+\frac{\delta}{4}\right)\left|u^{\prime}(x, t)\right|^{2}+\left(b_{1}+\frac{\delta C_{0}}{4}+\frac{\delta^{2} C_{0}}{4}\right)|\Delta u(x, t)|^{2} \\
& \left.+2 M\left(x, t,\|u(t)\|^{2}\right)|\nabla u(x, t)|^{2}\right] d x .
\end{aligned}
$$

Hence, denoting by $K_{2}=(1 / 2) \max \left\{1+\delta / 4, b_{1}+\delta C_{0} / 4+\delta^{2} C_{0} / 4,2\right\}$, we get

$$
\begin{aligned}
\frac{1}{K_{2}} H(t) \leq \int_{\Omega} & \left(\left|u^{\prime}(x, t)\right|^{2}+|\Delta u(x, t)|^{2}\right. \\
& \left.+M\left(x, t,\|u(t)\|^{2}\right)|\nabla u(x, t)|^{2}\right) d x .
\end{aligned}
$$

Thus, from (3.40) and (3.42), we can write

$$
\frac{d}{d t} H(t)+K_{3} H(t) \leq 0, \quad \text { where } K_{3}=\frac{K_{1}}{K_{2}} .
$$


As $u_{0}$ and $u_{1}$ belong to $H_{0}^{2}(\Omega)$ and $L^{2}(\Omega)$, respectively, then, from (3.43), we have

$$
H(t) \leq K_{4} \exp \left\{-K_{3} t\right\} \quad \forall t \geq 0 .
$$

From (3.44), we can conclude inequality (3.37). In fact, from the first inequality in (3.24), we obtain

$$
\begin{aligned}
2 H(t) \geq \frac{1}{2} \int_{\Omega}( & (x)\left|u^{\prime}(x, t)\right|^{2}+2 b(x)|\Delta u(x, t)|^{2} \\
& \left.+\frac{\delta^{2}}{4}|u(x, t)|^{2}+8 M\left(x, t,\|u(t)\|^{2}\right)|\nabla u(x, t)|^{2}\right) d x \quad \forall t \geq 0 .
\end{aligned}
$$

Hence, observing the definition of $E(t)$ in (3.38) and that $\left(\delta^{2} / 4\right)|u(x, t)|^{2} \geq 0$, we have

$$
2 H(t)>E(t) \quad \forall t \geq 0
$$

Finally, from (3.44) and (3.46), we get the desired proof of Theorem 3.3.

REMARK 3.4. As said before in Section 1, our main objective in this work is the study presented in Section 4. Thus, the results of Theorems 3.2 and 3.3 are sufficient to reach such objectives. However, it is not difficult to show that if we assume more restrictions, besides (3.2) and (3.3), about $a(x), b(x), \partial M / \partial t$, and $\partial M / \partial \lambda$, we obtain strong solutions for the boundary value problem (3.1).

\section{Noncylindrical domains}

4.1. Weak solutions. In the conditions of the notations of Section 2, we look for a real function $u=u(x, t)$ defined for all $(x, t) \in \hat{Q}$ solving the boundary value problem

$$
\begin{gathered}
a(x) u^{\prime \prime}(x, t)+\Delta(b(x) \Delta u(x, t)) \\
-\widehat{M}\left(x, t, \int_{\Omega_{t}}|\nabla u(x, t)|^{2} d x\right) \Delta u(x, t)+\delta u^{\prime}(x, t)=0 \quad \text { in } \hat{Q}, \\
u(x, t)=\frac{\partial u}{\partial v}(x, t)=0 \quad \text { on } \hat{\Sigma}, \\
u(x, 0)=u_{0}(x), \quad u^{\prime}(x, 0)=u_{1}(x) \quad \text { in } \Omega_{0} .
\end{gathered}
$$

We consider $\widehat{M}(x, t, \lambda)$ as the restriction of $M(x, t, \lambda)$ to $(x, t) \in \hat{Q}$ and $\lambda>0$, where $M(x, t, \lambda)$ is the function defined on the cylinder $Q=\Omega \times] 0, T$ [ which contains $\hat{Q}$.

As was said in Section 1, we are going to study the noncylindrical boundary value problem (4.1) by means of the penalty method idealized by Lions [8]. Thus, we assume certain hypotheses on the domain $\hat{Q}$ to apply the Lions' 
method. One condition is about the geometry of $\widehat{Q}$ and the other is on the regularity of its boundary $\hat{\Sigma}$. Therefore, we consider the sections $\Omega_{t}=\hat{Q} \cap\{t=s\}$ to be increasing with $s$. This condition means that if $s_{1} \leq s_{2}$, the projections of $\Omega_{s_{1}}, \Omega_{s_{2}}$ on the hyperplane $t=0$ are increasing, that is,

$$
\left.\left.\operatorname{proj}\right|_{t=0} \Omega_{s_{1}} \subseteq \operatorname{proj}\right|_{t=0} \Omega_{s_{2}} \text { if } s_{1} \leq s_{2} .
$$

As we have assumed in Section 2 that $\widehat{Q} \subset Q=\Omega \times] 0, T$, we need the following regularity condition:

(RC) if $v \in H_{0}^{2}(\Omega), v(x, t)=\partial v / \partial x_{i}=0$ a.e. in $\Omega-\Omega_{t}$, for $i=1,2, \ldots, n$ and for almost all $t \in] 0, T$, then $v \in H_{0}^{2}\left(\Omega_{t}\right)$.

Note that $v \in H_{0}^{2}\left(\Omega_{t}\right)$ is the restriction of $v$ to $\Omega_{t}$.

REMARK 4.1. In the original idea of Lions [8], he wanted to solve the boundary value problem for the operator

$$
u \rightarrow u^{\prime \prime}-\Delta u+|u|^{\rho} u
$$

in a noncylindrical domain $\hat{Q}$. Thus he needed a regularity similar to (RC) for $H_{0}^{1}(\Omega)$. Then, he supposed in [8] that if $v \in H_{0}^{1}(\Omega)$ and $v(x, t)=0$ in $\Omega-\Omega_{t}$, then $v \in H_{0}^{1}\left(\Omega_{t}\right)$. One example of this type of boundary is the case when $\Gamma_{t}=$ $\partial \Omega_{t}$ is of class $C^{1}$ almost everywhere in ]0,T[. In fact, in this case, we can apply trace theorem of order zero, that is, the trace $\gamma_{0}: H^{1}\left(\Omega-\Omega_{t}\right) \rightarrow H^{1 / 2}\left(\Gamma \cup \Gamma_{t}\right)$ (cf. [10,13]). Note that $\Gamma \cup \Gamma_{t}$ is the boundary of $\Omega-\Omega_{t}$. We also have, by trace theorem,

$$
\left\|\gamma_{0} v\right\|_{H^{1 / 2}\left(\Gamma \cup \Gamma_{t}\right)} \leq C\|v\|_{H^{1}\left(\Omega-\Omega_{t}\right)} .
$$

As $v=0$ almost everywhere in $\Omega-\Omega_{t}$, and $\gamma_{0} v=0$ on $\Gamma$ because $v \in H_{0}^{1}(\Omega)$, it follows from the inequality above that $\gamma_{0} v=0$ on $\Gamma_{t}$, which proves that $v \in H^{1}\left(\Omega_{t}\right)$ and $\gamma_{0} v=0$ on $\Gamma_{t}$, that is, $v \in H_{0}^{1}\left(\Omega_{t}\right)$. For the case $v \in H_{0}^{2}(\Omega)$,

$$
v(x, t)=\frac{\partial v}{\partial x_{i}}=0 \quad \text { a.e. in } \Omega-\Omega_{t},
$$

for $i=1,2, \ldots, n$. If we suppose $\Gamma_{t}$ to be of class $C^{2}$, we can employ the trace theorem of order two, that is, $\left\{\gamma_{0}, \gamma_{1}\right\}$, and obtain $v \in H_{0}^{2}\left(\Omega_{t}\right)$. Another method is to observe that $\partial u / \partial x_{i} \in H_{0}^{1}(\Omega)$ and $\partial u / \partial x_{i}=0$ almost everywhere in $\Omega-\Omega_{t}$ for $i=1,2, \ldots, n$ and apply the trace $\gamma_{0}$ as done above.

REMARK 4.2. Note that $a(x)$ and $b(x)$ in (4.1) are restrictions to $\hat{Q}$ of $a(x)$ and $b(x)$ defined in Section 3 satisfying hypothesis (3.2). Note also that $\widehat{M}(x, t, \lambda)$ satisfies hypothesis (3.3).

Due to the characteristics of the penalty method, it is only possible to obtain weak solutions for problem (4.1), which means solutions for (4.1) in the following sense. 
DEFINITION 4.3. A weak solution for the initial boundary value problem (4.1) is a real function $u=u(x, t)$, defined for $(x, t) \in \hat{Q}$ with

$$
u \in L^{2}\left(0, T ; H_{0}^{2}\left(\Omega_{t}\right)\right), \quad u^{\prime} \in L^{2}\left(0, T ; L^{2}\left(\Omega_{t}\right)\right), \quad \text { for } T>0,
$$

satisfying the integral identity

$$
\begin{aligned}
& -\int_{0}^{T} \int_{\Omega_{t}} a(x) u^{\prime}(x, t) \phi^{\prime}(x, t) d x d t \\
& \quad+\int_{0}^{T} \int_{\Omega_{t}} b(x) \Delta u(x, t) \Delta \phi(x, t) d x d t \\
& \quad+\int_{0}^{T} \int_{\Omega_{t}} M\left(x, t, \int_{\Omega_{t}}|\nabla u(x, t)|^{2} d x\right) \nabla u(x, t) \cdot \nabla \phi(x, t) d x d t \\
& \quad+\int_{0}^{T} \int_{\Omega_{t}}\left[\nabla_{x} M\left(x, t, \int_{\Omega_{t}}|\nabla u(x, t)|^{2} d x\right) \cdot \nabla u(x, t)\right] \phi(x, t) d x d t \\
& \quad+\delta \int_{0}^{T} \int_{\Omega_{t}} u^{\prime}(x, t) \phi(x, t) d x d t=0 \quad \forall \phi \text { such that } \\
& \phi \in L^{2}\left(0, T ; H_{0}^{2}\left(\Omega_{t}\right)\right), \quad \phi^{\prime} \in L^{2}\left(0, T ; L^{2}\left(\Omega_{t}\right)\right) \quad \text { with } \phi(x, 0)=\phi(x, T)=0,
\end{aligned}
$$

and the initial conditions

$$
u(x, 0)=u_{0}(x), \quad u^{\prime}(x, 0)=u_{1}(x) \text { for } x \in \Omega_{0}
$$

THEOREM 4.4. Suppose $u_{0} \in H_{0}^{2}\left(\Omega_{0}\right), u_{1} \in L^{2}\left(\Omega_{0}\right)$, and

$$
C_{6} H^{p}(0)<\frac{\delta b_{0}}{10}
$$

where the function $H(t)$ and the constant $C_{6}$ are defined in (3.24) and (3.36), respectively. Then there exists at least one function $u: \hat{Q} \rightarrow \mathbb{R}$ as a weak solution of the boundary value problem (4.1) in the sense of Definition 4.3.

Proof. We employ the penalty method to transform the noncylindrical problem in $\hat{Q}$ into a cylindrical problem in $Q$ and then we use the FaedoGalerkin method as done in Section 3 with the necessary modifications.

Defining the function

$$
\chi(x, t)= \begin{cases}1 & \text { in } \Omega \times] 0, T\left[\backslash \hat{Q} \cup\left\{\Omega_{0} \times\{0\}\right\},\right. \\ 0 & \text { in } \hat{Q} \cup\left\{\Omega_{0} \times\{0\}\right\},\end{cases}
$$

we have $x \in L^{\infty}(\Omega \times] 0, T[)$.

Denoting by $\tilde{u}_{0}, \tilde{u}_{1}$ the extension of $u_{0}, u_{1}$ to $\Omega$ defined zero outside of $\Omega-\Omega_{0}$, we have $\tilde{u}_{0} \in H_{0}^{2}(\Omega)$ and $\tilde{u}_{1} \in L^{2}(\Omega)$. Thus, we define the following penalized problem. 
Given $\epsilon>0$, we look for $u_{\epsilon}=u_{\epsilon}(x, t)$ in the class

$$
u_{\epsilon} \in L^{2}\left(0, T ; H_{0}^{2}(\Omega)\right), \quad u_{\epsilon}^{\prime} \in L^{2}\left(0, T ; L^{2}(\Omega)\right) \text { for } T>0,
$$

satisfying the integral identity

$$
\begin{aligned}
-\int_{Q} a & (x) u_{\epsilon}^{\prime}(x, t) \phi^{\prime}(x, t) d x d t+\int_{Q} b(x) \Delta u_{\epsilon}(x, t) \Delta \phi(x, t) d x d t \\
& +\int_{Q} M\left(x, t,\left\|u_{\epsilon}(t)\right\|^{2}\right) \nabla u_{\epsilon}(x, t) \cdot \nabla \phi(x, t) d x d t \\
& +\int_{Q}\left[\nabla_{x} M\left(x, t,\left\|u_{\epsilon}(t)\right\|^{2}\right) \cdot \nabla u_{\epsilon}(x, t)\right] \phi(x, t) d x d t \\
& +\delta \int_{Q} u_{\epsilon}^{\prime}(x, t) \phi(x, t) d x d t \\
& +\frac{1}{\epsilon} \int_{Q} x(x, t) u_{\epsilon}^{\prime}(x, t) \phi(x, t) d x d t \\
& +\frac{1}{\epsilon} \int_{Q} x(x, t) \nabla u_{\epsilon}(x, t) \cdot \nabla \phi(x, t) d x d t=0
\end{aligned}
$$

for all $\phi$ such that $\phi \in L^{2}\left(0, T ; H_{0}^{2}\left(\Omega_{t}\right)\right), \phi^{\prime} \in L^{2}\left(0, T ; L^{2}\left(\Omega_{t}\right)\right)$ with $\phi(x, 0)=$ $\phi(x, T)=0$, and the initial conditions

$$
u_{\epsilon}(x, 0)=\tilde{u}_{0}(x), \quad u_{\epsilon}^{\prime}(x, 0)=\tilde{u}_{1}(x) \text { for } x \in \Omega_{0} .
$$

The penalized problem is cylindrical. Then we are in the case of Section 3. In fact, we consider a Hilbertian basis with vectors $\left(w_{j}\right)_{j \in \mathbb{N}}$ in $H_{0}^{2}(\Omega)$ (cf. [1]), such that $w_{1}=\tilde{u}_{0}$. By using the same notation of Section 3 we look for $u_{\epsilon m} \in V_{m}$, for $\epsilon$ fixed, such that

$$
\begin{aligned}
\int_{\Omega} a(x) & u_{\epsilon m}^{\prime \prime}(x, t) w(x) d x+\int_{\Omega} b(x) \Delta u_{\epsilon m}(x, t) \Delta w(x) d x \\
& +\int_{\Omega} M\left(x, t,\left\|u_{\epsilon m}(t)\right\|^{2}\right) \nabla u_{\epsilon m}(x, t) \cdot \nabla w(x) d x \\
& +\int_{\Omega}\left[\nabla x M\left(x, t,\left\|u_{\epsilon m}(t)\right\|^{2}\right) \cdot \nabla u_{\epsilon m}(x, t)\right] w(x) d x \\
+ & \delta \int_{\Omega} u_{\epsilon m}^{\prime}(x, t) w(x) d x+\frac{1}{\epsilon} \int_{\Omega} x(x, t) u_{\epsilon m}^{\prime}(x, t) w(x) d x \\
+ & \frac{1}{\epsilon} \int_{\Omega} x(x, t) \nabla u_{\epsilon m}(x, t) \cdot \nabla w(x) d x=0 \quad \forall w \in V_{m}, \\
& u_{\epsilon m}(x, 0)=\tilde{u}_{0}, \quad u_{\epsilon m}^{\prime}(x, 0)=u_{1 m} \rightarrow \tilde{u}_{1} \quad \text { in } L^{2}(\Omega) .
\end{aligned}
$$

The initial value problem (4.14) and (4.15) has a local solution $u_{\epsilon m}$ defined on the interval $\left[0, t_{m}\right.$ [ for each $\epsilon>0$ fixed. The extension to the whole interval 
$[0, T$ for each $T>0$ depends on the estimates that we will find below. These estimates are also sufficient to pass to the limits as $m \rightarrow \infty$ and $\epsilon \rightarrow 0$. The computations are those that were done in Section 3, however, we will make a summary. In fact, initially, we set both $w=u_{\epsilon m}^{\prime}(x, t)$ and $w=u_{\epsilon m}(x, t)$ into (4.14). After that, multiplying the second estimate, which comes from the substitution of $w$ by $u_{\epsilon m}(x, t)$, by $\delta / 4$ and proceeding adding to the first one yields

$$
\begin{aligned}
\frac{d}{d t} H(t) & +\left|\Delta u_{\epsilon m}(t)\right|^{2}\left(\frac{\delta b_{0}}{8}-C_{3}\left|\Delta u_{\epsilon m}(t)\right|^{2 p}\right. \\
& \left.\quad-C_{4}\left|\Delta u_{\epsilon m}(t)\right|^{2 p-1}\left|u_{\epsilon m}^{\prime}(t)\right|-\frac{C_{5} \delta}{4}\left|\Delta u_{\epsilon m}(t)\right|^{2 p}\right) \\
+ & \frac{1}{\epsilon} \int_{\Omega} x(x, t)\left|u_{\epsilon m}^{\prime}(x, t)\right|^{2} d x \\
+ & \frac{1}{\epsilon} \int_{\Omega} x(x, t) u_{\epsilon m}^{\prime}(x, t) u_{\epsilon m}(x, t) d x \\
+ & \frac{1}{\epsilon} \int_{\Omega} x(x, t) \nabla u_{\epsilon m}^{\prime}(x, t) \cdot \nabla u_{\epsilon m}(x, t) d x \\
+ & \frac{1}{\epsilon} \int_{\Omega} x(x, t)\left|\nabla u_{\epsilon m}(x, t)\right|^{2} d x+\frac{\delta b_{0}}{8}\left|\Delta u_{\epsilon m}(t)\right|^{2} \\
+ & \frac{3 \delta}{4}\left|u_{\epsilon m}^{\prime}(t)\right|^{2}+\frac{\delta}{4} \int_{\Omega} M\left(x, t,\left\|u_{\epsilon m}(t)\right\|^{2}\right)\left|\nabla u_{\epsilon m}(x, t)\right|^{2} d x \leq 0,
\end{aligned}
$$

where $H(t)=H\left(u_{\epsilon m}(t)\right)$ is defined in (3.24) with $u_{\epsilon m}(t)$ in the place of $u(t)$, and hence, we have from (3.26) that

$$
\begin{aligned}
H(t) \geq \frac{1}{4} \int_{\Omega}( & a(x)\left|u_{\epsilon m}^{\prime}(x, t)\right|^{2}+\frac{1}{2} b(x)\left|\Delta u_{\epsilon m}(x, t)\right|^{2} \\
& \left.+\frac{\delta^{2}}{16}\left|u_{\epsilon m}(x, t)\right|^{2}+M\left(x, t,\left\|u_{\epsilon m}(t)\right\|^{2}\right)\left|\nabla u_{\epsilon m}(x, t)\right|^{2}\right) d x \geq 0 .
\end{aligned}
$$

As in Section 3, we define

$$
\gamma(t)=C_{3}\left|\Delta u_{\epsilon m}(t)\right|^{2 p}+C_{4}\left|\Delta u_{\epsilon m}(t)\right|^{2 p-1}\left|u_{\epsilon m}^{\prime}(t)\right|+\frac{C_{5} \delta}{4}\left|\Delta u_{\epsilon m}(t)\right|^{2 p} .
$$

As in (3.34) and hypothesis (4.9), we can write

$$
\gamma(t) \leq C_{6} H^{p}(t), \quad C_{6} H^{p}(0)<\frac{\delta b_{0}}{10} .
$$


Thus, by the same way of Section 3, we obtain from (4.16) and the hypothesis on initial data $\tilde{u}_{0}, \tilde{u}_{1}$ that

$$
\begin{aligned}
\frac{d}{d t} H(t) & +\frac{1}{\epsilon} \int_{\Omega} x(x, t)\left|u_{\epsilon m}^{\prime}(x, t)\right|^{2} d x \\
& +\frac{1}{\epsilon} \int_{\Omega} x(x, t) u_{\epsilon m}^{\prime}(x, t) u_{\epsilon m}(x, t) d x \\
& +\frac{1}{\epsilon} \int_{\Omega} x(x, t) \nabla u_{\epsilon m}^{\prime}(x, t) \cdot \nabla u_{\epsilon m}(x, t) d x \\
& +\frac{1}{\epsilon} \int_{\Omega} x(x, t)\left|\nabla u_{\epsilon m}(x, t)\right|^{2} d x \leq 0 \quad \forall t \geq 0 .
\end{aligned}
$$

By using the idea of Nakao and Narazaki [14] or Rabello [5], we have

$$
\begin{gathered}
\int_{0}^{t} \int_{\Omega} \chi(x, t) u_{\epsilon m}^{\prime}(x, t) u_{\epsilon m}(x, t) d x d t \\
\geq\left|\chi(t) u_{\epsilon m}(t)\right|^{2}-\left|\chi \tilde{u}_{0}\right|^{2}, \\
\int_{0}^{t} \int_{\Omega} \chi(x, t) \nabla u_{\epsilon m}^{\prime}(x, t) \cdot \nabla u_{\epsilon m}(x, t) d x d t \\
\geq\left|\chi(t) \nabla u_{\epsilon m}(t)\right|^{2}-\left|\chi \nabla \tilde{u}_{0}\right|^{2} .
\end{gathered}
$$

Observe that, from the definition of $\tilde{u}_{0}$ and $\chi(x, t)$, we have

$$
\begin{gathered}
\left|\chi \tilde{u}_{0}\right|^{2}=\int_{\Omega} \chi(x, 0) \tilde{u}_{0}(x) d x=0, \\
\left|\chi \nabla \tilde{u}_{0}\right|^{2}=\int_{\Omega} \chi(x, 0) \nabla \tilde{u}_{0}(x) d x=0 .
\end{gathered}
$$

Thus, the integrals in (4.21) are positives. Then, from (4.20), integrating from 0 to $t$, we obtain

$$
H(t) \leq H(0) \quad \forall t>0, \quad \epsilon>0 .
$$

From (4.23), we extract a subsequence, still represented by $\left(u_{\epsilon m}\right)_{m \in \mathbb{N}}$, such that

$$
\begin{aligned}
& u_{\epsilon m}-u_{\epsilon} \quad \text { weak star in } L^{\infty}\left(0, T ; H_{0}^{2}(\Omega)\right) \text { as } m \longrightarrow \infty, \\
& u_{\epsilon m}^{\prime}-u_{\epsilon}^{\prime} \quad \text { weak star in } L^{\infty}\left(0, T ; L^{2}(\Omega)\right) \text { as } m \longrightarrow \infty .
\end{aligned}
$$

From (4.24), (4.25), and Aubin-Lions theorem on compactness (cf. Lions [9]), we obtain a subsequence, still represented by $\left(u_{\epsilon m}\right)_{m \in \mathbb{N}}$, such that

$$
u_{\epsilon m} \longrightarrow u_{\epsilon} \text { strongly in } L^{2}\left(0, T ; H_{0}^{1}(\Omega)\right) \text { as } m \longrightarrow \infty .
$$

We also have

$$
\begin{array}{cl}
\chi u_{\epsilon m}^{\prime}-\chi u_{\epsilon}^{\prime} & \text { weak star in } L^{\infty}\left(0, T ; L^{2}(\Omega)\right) \text { as } m \rightarrow \infty, \\
\chi \nabla u_{\epsilon m}-\chi \nabla u_{\epsilon} & \text { weak star in } L^{\infty}\left(0, T ; L^{2}(\Omega)\right) \text { as } m \rightarrow \infty .
\end{array}
$$


Setting $v \in H_{0}^{2}(\Omega)$ into (4.14), multiplying by $\theta \in \mathscr{D}(0, T)$, taking the limit as $m \rightarrow \infty$, and observing the convergence from (4.24) to (4.28), we conclude that $u_{\epsilon}$ is a weak solution of the penalized problem (4.12) and (4.13).

The next step is the study of the penalized problem (4.12) and (4.13) as $\epsilon \rightarrow 0$ in order to conclude the proof of Theorem 4.4. In fact, from convergence (4.24), (4.25), (4.26), and Banach-Steinhaus theorem, we obtain a net $\left(u_{\epsilon}\right)_{0<\epsilon<1}$ and a function $\omega: Q \rightarrow \mathbb{R}$ satisfying

$$
\begin{gathered}
u_{\epsilon} \rightarrow \omega \quad \text { weak star in } L^{\infty}\left(0, T ; H_{0}^{2}(\Omega)\right) \text { as } \epsilon \longrightarrow 0, \\
u_{\epsilon}^{\prime} \rightarrow \omega^{\prime} \quad \text { weak star in } L^{\infty}\left(0, T ; L^{2}(\Omega)\right) \text { as } \epsilon \longrightarrow 0, \\
u_{\epsilon} \rightarrow \omega \quad \text { strongly in } L^{2}\left(0, T ; H_{0}^{1}(\Omega)\right) \text { as } \epsilon \longrightarrow 0 \text { and a.e. in } Q .
\end{gathered}
$$

From (4.21), we get from (4.20) that

$$
\begin{gathered}
\int_{\Omega \times] 0, T[} x\left|u_{\epsilon m}^{\prime}(x, t)\right|^{2} d x d t \leq \epsilon C, \\
\int_{\Omega \times] 0, T[} x\left|\nabla u_{\epsilon m}(x, t)\right|^{2} d x d t \leq \epsilon C,
\end{gathered}
$$

where $C$ is a real positive constant independent of $\epsilon$ and $m$. Thus, from (4.27) and Banach-Steinhaus theorem, we have $\left\|\chi u_{\epsilon}^{\prime}\right\|_{L^{2}\left(0, T ; L^{2}(\Omega)\right)}<\epsilon C$. Hence we affirm that $\chi u_{\epsilon}^{\prime}$ converges weakly in $L^{2}\left(0, T ; L^{2}(\Omega)\right)$ to zero. Therefore, from (4.30), we get

$$
\int_{0}^{T} \int_{\Omega} x(x, t)\left|\omega^{\prime}(x, t)\right|^{2} d x d t=0 .
$$

By similar argument, we obtain from (4.29) that

$$
\int_{0}^{T} \int_{\Omega} x(x, t)|\nabla \omega(x, t)|^{2} d x d t=0 .
$$

From (4.33), we have

$$
\left.\chi(x, t) \omega^{\prime}(x, t)=0 \quad \text { a.e. in } \Omega \times\right] 0, T[=Q,
$$

and then,

$$
\omega^{\prime}(x, t)=0 \quad \text { in } Q-\hat{Q} \cup \Omega_{0} \times\{0\} .
$$

Since $\hat{Q}$ is increasing, we have

$$
\int_{0}^{t} \omega^{\prime}(x, s) d s=0 \quad \forall 0<t<T, x \in \Omega-\Omega_{0} .
$$

But, as $w(x, 0)=\tilde{u}_{0}(x)=0$ in $\Omega-\Omega_{0}$, by virtue of $\omega$ as a solution in $Q$, then

$$
\omega(x, t)=0 \quad \text { a.e. in } \Omega-\Omega_{t} \text { for } 0<t<T .
$$


From (4.34) and by a similar way, we get

$$
\chi(x, t)|\nabla \omega(x, t)|=0 \quad \text { in } Q-\hat{Q} \cup \Omega_{0} \times\{0\},
$$

and then it implies that

$$
\frac{\partial \omega(x, t)}{\partial x_{i}}=0 \quad \text { a.e. } \Omega-\Omega_{t} \text { for } 0<t<T \text {. }
$$

From (4.38), (4.40), and if $u$ is the restriction of $\omega$ to $\hat{Q}$, then we get by using (4.29),

$$
u \text { belongs to } L^{\infty}\left(0, T ; H_{0}^{2}\left(\Omega_{t}\right)\right) \text {, }
$$

where we have used to obtain (4.41) the hypothesis of regularity on $\hat{Q}$ established in (RC).

In a similar way, if $u^{\prime}$ is the restriction of $\omega^{\prime}$ to $\hat{Q}$, then we get by using (4.30)

$$
u^{\prime} \text { belongs to } L^{\infty}\left(0, T ; L^{2}\left(\Omega_{t}\right)\right) \text {. }
$$

Thus, by restriction to $\hat{Q}$ of the penalized equation (4.12), we obtain

$$
\begin{aligned}
-\int_{\hat{Q}} a(x) & \hat{u}_{\epsilon}^{\prime}(x, t) \phi^{\prime}(x, t) d x d t \\
& +\int_{\hat{Q}} b(x) \Delta \hat{u}_{\epsilon}(x, t) \Delta \phi(x, t) d x d t \\
& +\int_{\hat{Q}} \widehat{M}\left(x, t,\left\|\hat{u}_{\epsilon}(t)\right\|^{2}\right) \nabla \hat{u}_{\epsilon}(x, t) \cdot \nabla \phi(x, t) d x d t \\
& +\int_{\hat{Q}}\left[\nabla_{x} \widehat{M}\left(x, t,\left\|\hat{u}_{\epsilon}(t)\right\|^{2}\right) \cdot \nabla \hat{u}_{\epsilon}(x, t)\right] \phi(x, t) d x d t \\
& +\delta \int_{\hat{Q}} \hat{u}_{\epsilon}^{\prime}(x, t) \phi(x, t) d x d t=0
\end{aligned}
$$

for all $\phi$ such that $\phi \in L^{2}\left(0, T ; H_{0}^{2}\left(\Omega_{t}\right)\right), \phi^{\prime} \in L^{2}\left(0, T ; L^{2}\left(\Omega_{t}\right)\right)$ with $\phi(x, 0)=$ $\phi(x, T)=0$.

Thus, $\widehat{M}(x, t, \lambda)$ is the restriction of $M(x, t, \lambda)$ to $\hat{Q}$ for $\lambda>0$ and $u$ is the restriction of $\omega$ to $\hat{Q}$. From the convergence (4.29), (4.30), (4.31), and passing to the limit in (4.43) as $\epsilon \rightarrow 0$, it follows that $u$ is a solution of (4.1) in the sense of Definition 4.3. And this way, the proof of Theorem 4.3 is concluded.

4.2. Exponential decay. By using the same method employed in Section 3.2, we obtain the exponential decay for the energy associated with the system (4.1) as $t \rightarrow+\infty$, that is, the exponential decay within of the noncylindrical domain. 
ACKNOWLEDGMENT. This work was sponsored partially by PROPP-UFF.

\section{REFERENCES}

[1] H. Brezis, Analyse Fonctionelle (Théorie et Applications), Dunod, Paris, 1999 (French).

[2] J. Cooper and C. Bardos, A nonlinear wave equation in a time dependent domain, J. Math. Anal. Appl. 42 (1973), 29-60.

[3] J. Cooper and L. A. Medeiros, The Cauchy problem for non linear wave equations in domains with moving boundary, Ann. Scuola Norm. Sup. Pisa (3) 26 (1972), 829-838.

[4] M. Hosoya and Y. Yamada, On some nonlinear wave equations. II. Global existence and energy decay of solutions, J. Fac. Sci. Univ. Tokyo Sect. IA Math. 38 (1991), no. 2, 239-250.

[5] T. N. Rabello, Decay of solutions of a nonlinear hyperbolic system in noncylindrical domain, Int. J. Math. Math. Sci. 17 (1994), no. 3, 561-570.

[6] W. A. Strauss, On weak solutions of semi-linear hyperbolic equations, An. Acad. Brasil. Ci. 42 (1970), 645-651.

[7] A. Inoue, Sur $\square u+u^{3}=f$ dans un domaine noncylindrique, J. Math. Anal. Appl. 46 (1974), 777-819 (French).

[8] J.-L. Lions, Une remarque sur les problèmes d'évolution non linéaires dans des domaines non cylindriques, Rev. Roumaine Math. Pures Appl. 9 (1964), 1118 (French).

[9]__ Quelques méthodes de résolution des problèmes aux limites non linéaires, Dunod, Paris, 1969 (French).

[10] J.-L. Lions and E. Magenes, Problèmes aux limites non homogènes et applications. Vol. 1, Travaux et Recherches Mathématiques, no. 17, Dunod, Paris, 1968 (French).

[11] L. A. Medeiros, Nonlinear wave equations in domains with variable boundary, Arch. Rational Mech. Anal. 47 (1972), 47-58.

[12] _ Semilinear wave equations, Partial Differential Equations and Related Topics (Program, Tulane Univ., New Orleans, La, 1974) (J. A. Goldstein, ed.), Lecture Notes in Math., vol. 446, Springer, Berlin, 1975, pp. 329-354.

[13] L. A. Medeiros and M. Milla Miranda, Espaços de Sobolev (Introdução aos problemas elíticos não homogeneos), Institudo de Matemática, Universidade Federal do Rio de Janeiro, Rio de Janeiro, 2000 (Portuguese).

[14] M. Nakao and T. Narazaki, Existence and decay of solutions of some nonlinear wave equations in noncylindrical domains, Math. Rep. Kyushu Univ. 11 (1978), no. 2, 117-125.

J. Límaco: IM-GMA, Universidade Federal Fluminense, Niteri, RJ, Brazil

E-mail address: juanbrj@hotmai 1.com.br

H. R. Clark: IM-GAN, Universidade Federal Fluminense, Niteri, RJ, Brazil

E-mail address: ganhrc@vm.uff.br

L. A. Medeiros: Universidade Federal do Rio de Janeiro, IM, Rio de Janeiro, RJ, Brazil

E-mail address: 1medeiros@abc.org.br 


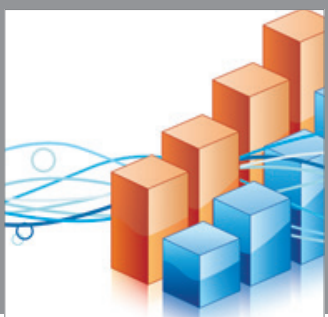

Advances in

Operations Research

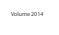

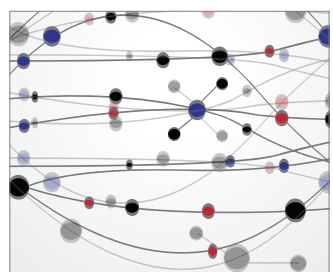

\section{The Scientific} World Journal
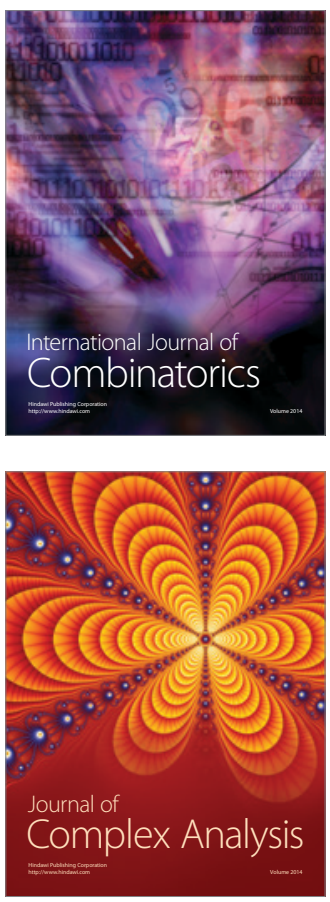

International Journal of

Mathematics and

Mathematical

Sciences
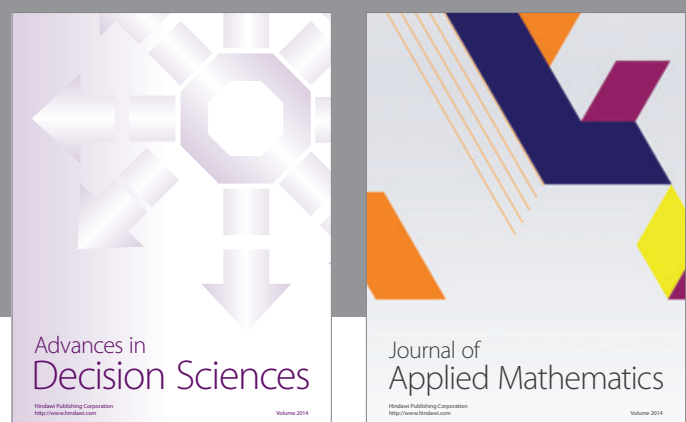

Journal of

Applied Mathematics
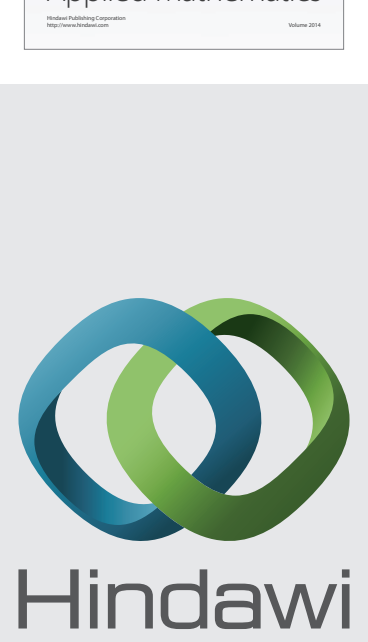

Submit your manuscripts at http://www.hindawi.com
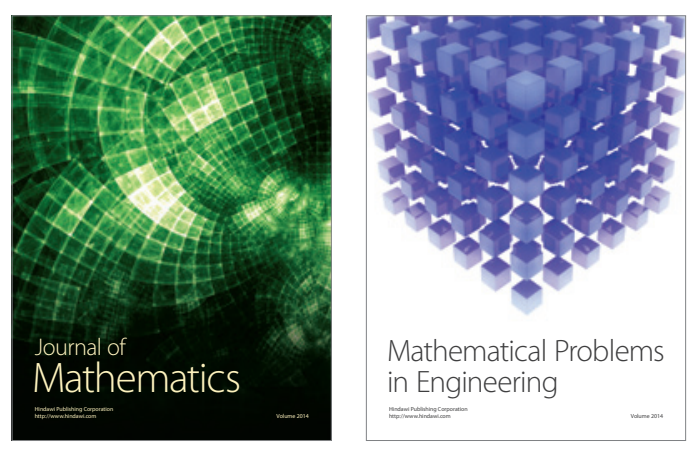

Mathematical Problems in Engineering
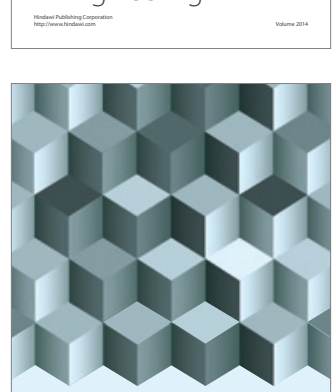

Journal of

Function Spaces
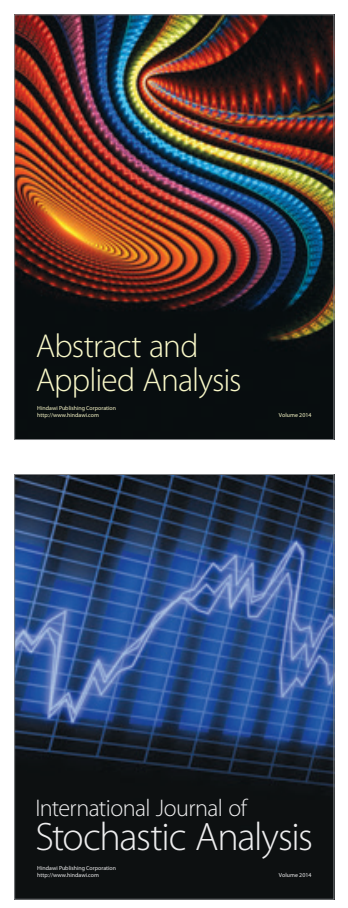

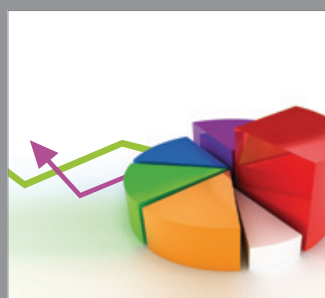

ournal of

Probability and Statistics

Promensencen
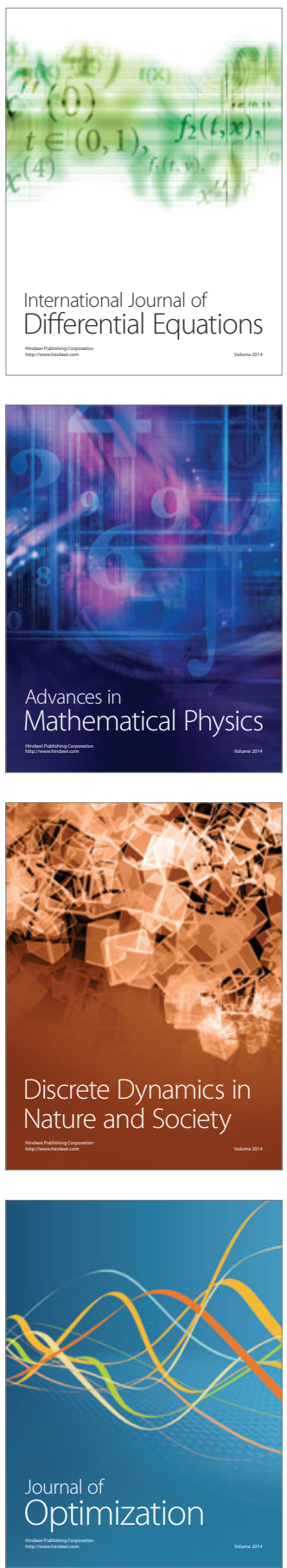